\title{
k-diskcyclic operators on Banach spaces
}

\begin{abstract}
In this paper, we define and study new classes of operators on complex Banach spaces, which we call k-diskcyclic. We use these operators to show that the direct sum of a diskcyclic operator with it self $\mathrm{k}$ times ( $\mathrm{k}$ Ó2) does not need to be diskcyclic. However, we show that under certain conditions the latter statement holds true. In particular, we show that an operator $\mathrm{T}$ satisfies the diskcyclic criterion if and only if $\mathrm{T}$ is $\mathrm{k}$-diskcyclic.
\end{abstract}

Keyword: Banach spaces; k-diskcyclic 